ARTIGO ORIGINAL

\title{
A dominância de membros interfere no equilíbrio de força muscular do joelho de corredores recreacionais?
}

\author{
Does the limb dominance interfere with knee muscle strength balance in recreational \\ runners?
}

Murilo Pires Neves ${ }^{1}$, Cristiano Sena da Conceição², Mansueto Gomes Neto ${ }^{3 *}$

\begin{abstract}
${ }^{1}$ Mestrando em Processos Interativos de Órgãos e Sistemas pela Universidade Federal da Bahia.; ${ }^{2}$ Docente Permanente do Programa de Pós-Graduação em Processos Interativos de Órgãos e Sistemas da Universidade Federal da Bahia.; ${ }^{3}$ Docente Permanente do Programa de Pós-Graduação em Processos Interativos de Órgãos e

Sistemas da Universidade Federal da Bahia
\end{abstract}

\begin{abstract}
Resumo
Introdução: cerca de $5 \%$ da população brasileira pratica corrida de rua, e a falta de preparo físico das pessoas para esse esporte contribui para o aumento no índice de lesões, sendo o joelho uma das articulações mais acometidas. Sabendo que os parâmetros musculares interferem diretamente no desempenho e que a destreza de membros pode suscitar inferências relativas ao aumento de força no membro dominante, torna-se importante avaliar a força e a relação de equilíbrio muscular do joelho destes atletas tanto no membro dominante quanto no contralateral. Objetivo: verificar o equilíbrio muscular dos extensores e flexores de joelho dominante e não dominante em corredores recreacionais. Metodologia: foram incluídos 111 indivíduos com idade entre 18 e 65 anos, de ambos os sexos, praticantes de corrida há pelo menos 4 meses contínuos, sem histórico de lesão nos últimos 3 meses. Os participantes foram entrevistados e encaminhados para a coleta da força muscular isocinética dos grupos extensor e flexor do joelho com protocolo de $60 \% \mathrm{~s}, 180^{\circ} / \mathrm{s}$ e $300^{\circ} \mathrm{s}$. As variáveis de interesse estudadas foram: membro dominante, tempo de prática de corrida, torque máximo, trabalho total e potência. Foram calculados os Índices de Deficiência Muscular (IDM) sendo admitido como referencial de equilíbrio até $10 \%$ na diferença entre os membros. A pesquisa foi aprovada pelo Comitê de Ética em Pesquisa do Instituto de Ciências da Saúde da Universidade Federal da Bahia, sob parecer de no 2.621.166. Resultados: o IDM indicou que 54,1\% dos extensores de joelho estavam equilibrados e que $55,9 \%$ dos flexores de joelho encontravam-se numa relação de desequilíbrio muscular. Conclusão: a dominância de membros não é um fator ligado ao desequilíbrio de forças no membro inferior. Este achado de desequilíbrio de força entre joelhos pode estar ligado à predisposição de lesão e deve orientar as equipes multiprofissionais de saúde a definir um trabalho preventivo de treinamento muscular e esportivo.
\end{abstract}

Palavras-chave: Equilíbrio Muscular. Extensores e Flexores do Joelho. Dinamometria Isocinética. Corredores.

\begin{abstract}
Introduction: about 5\% of the Brazilian population practices street running and the lack of physical fitness for this sport contributes to the increase in the injury rate, with the knee being one of the most affected joints. Knowing that muscle parameters directly interfere performance and that limb dexterity may lead to inferences regarding strength increase in the dominant limb, it is important to evaluate the strength and balance ratio of these athletes' knee in both dominant and contralateral limbs. Objective: to verify muscle balance of dominant and non-dominant knee extensors and flexors in recreational runners. Methodology: 111 individuals aged between 18 and 65 years old, male and female, who have been running for at least 4 continuous months, with no history of injury in the last 3 months, were included. Participants were interviewed and referred for the collection of isokinetic muscle strength of the knee extensor and flexor groups with a protocol of $60 \% \mathrm{~s}, 180 \% \mathrm{~s}$ and $300 \%$ s. The variables of interest studied were: dominant limb, running practice time, maximum torque, total work and power. Muscle deficiency indices (MDI) were calculated and accepted as a balance reference up to $10 \%$ in the difference between the limbs. The research was approved by the Research Ethics Committee of the Institute of Health Sciences of the Federal University of Bahia, under technical advice no 2.621.166. Results: the MDI indicated that $54.1 \%$ of the knee extensors were balanced and $55.9 \%$ of the knee flexors were in a muscular imbalance ratio. Conclusion: limb dominance is not a factor related to lower limb imbalance of forces. This finding of strength imbalance between knees may be associated to injury predisposition and should guide multidisciplinary health teams to define preventive work on muscle and sports training. Keywords: Muscle balance. Knee Extensors and Flexors. Isokinetic Dynamometry. Runners.
\end{abstract}

Correspondente/Corresponding: *Mansueto Gomes-Neto - Departamento de Fisioterapia, Instituto de Ciências da Saúde, Curso de Fisioterapia, Universidade Federal da Bahia (UFBA) - End: Av. Reitor Miguel Calmon, s/n-Vale do Canela, 40110-100 Salvador, BA - Tel: (71) 99918-8277 - Email: mansueto.neto@ufba.br

\section{INTRODUÇÃO}

Atualmente, cerca de $5 \%$ da população brasileira pratica corrida de rua, o que equivale a aproximadamente 10 milhões de corredores. A busca por hábitos de vida mais saudáveis e por melhora de condicionamento físico, unidos ao baixo custo e à fácil execução, definem os principais motivos para a procura da corrida como esporte. Em 
contrapartida, a prática não orientada e a falta de preparo das pessoas para esse esporte contribuem para o aumento no índice de lesões (ALVES; DUARTE JUNIOR, 2014).

Neste cenário, três fatores de risco para lesões são constantemente atribuídos ao público praticante de corrida: os relacionados ao treinamento, os anatômicos e os biomecânicos (HRELJAC, 2005; LOPES et al., 2012; SARAGIOTTO et al., 2014; STRAKOWSKI; JAMIL, 2006). Nos últimos anos, o estudo sistemático da biomecânica da marcha e corrida vem sendo alvo constante de diferentes centros de pesquisas esportivas, buscando melhorar a compreensão das lesões de membro inferior. Grande parte dos estudos apontam para um funcionamento desarmonioso das articulações e ativações musculares, influenciando negativamente na realização de um padrão biomecânico adequado (FREDERICSON; MISRA, 2007; HINO et al., 2009; LEPPÄNEN et al., 2014; SOUZA et al., 2011).

A influência do desequilíbrio muscular dos membros inferiores para a incidência de lesões em corredores é uma variável já explorada em alguns estudos. Concomitante, o mecanismo de repetição, associado à alta demanda mecânica durante a corrida, leva a um desenvolvimento muscular específico. E é justamente essa especificidade durante o treinamento muscular que poderá desencadear desequilíbrios sobre as articulações, gerando uma mecânica ou postura alteradas e predispondo os atletas a lesões e/ou redução do desempenho esportivo (SIQUEIRA et al., 2002). Isso foi relatado em um estudo recente (SARAGIOTTO et al., 2016) que apontou associação entre o surgimento de lesões e o desequilíbrio de força entre músculos flexores e extensores de joelho em corredores.

Portanto, sabendo que os parâmetros musculares interferem diretamente no desempenho do atleta e na sua predisposição a lesões, torna-se importante avaliar a força e a relação de equilíbrio muscular destes atletas. Para este fim, o método utilizado como padrão-ouro é a dinamometria isocinética. Nesse método, o indivíduo faz um contínuo esforço muscular máximo ou submáximo, em velocidade angular predefinida e constante, que se acomoda à resistência do aparelho, trazendo correspondência exata entre o torque aplicado e a resistência (DANNESKIOLD-SAMS $\varnothing$ E et al., 2009; DVIR, 2002; PONCE et al., 2013; SARAGIOTTO et al., 2016; XAVEROVA et al., 2015).

Diante da ascensão da prática de corrida recreativa e da sua influência no risco de lesões, percebe-se a necessidade de estudar mais profundamente o delineamento de equilíbrio muscular dos membros inferiores. Devido ao relevante papel dos grupamentos extensor e flexor dos joelhos, sua avaliação tornou-se o foco principal desse estudo. A avaliação isocinética do joelho, a partir dessa perspectiva investigativa de equilíbrio muscular, poderá trazer real benefício para nortear o desempenho esportivo. Esse aspecto certamente irá orientar e melhor indicar a tomada de decisão na prática clínica. Portanto, o objetivo do presente estudo consiste em descrever o equilíbrio muscular dos extensores e flexores de joelho dominante e não dominante em corredores recreacionais.

\section{METODOLOGIA}

O estudo foi conduzido no laboratório da sala 101 no Departamento do Curso de Fisioterapia do Instituto de Ciências da Saúde da Universidade Federal da Bahia, situada em Salvador, Bahia. Tratou-se de um estudo de corte transversal, observacional e descritivo, com base em dados primários coletados de corredores recrutados em assessorias esportivas ou clubes de corrida da cidade de Salvador.

Foram incluídos 111 indivíduos com idades entre 18 e 65 anos, praticantes de corrida há pelo menos 4 meses contínuos por pelo menos 1 vez por semana, sem histórico de lesão nos últimos 3 meses e que aceitaram participar do estudo assinando o Termo de Consentimento Livre e Esclarecido - TCLE. Não foram incluídos sujeitos que apresentaram histórico de cirurgias na extremidade inferior ou que eram praticantes de corrida descalça. $O$ trabalho obedeceu aos critérios de ética em pesquisa com seres humanos, tendo sido aprovado pelo Comitê de Ética em Pesquisa do Instituto de Ciências da Saúde da Universidade Federal da Bahia, sob parecer de no 2.621.166.

Os participantes foram entrevistados para coleta de dados referentes às informações sociodemográficas, antropométricas e de tempo de prática de corrida. $\mathrm{Na}$ sequência, foram encaminhados para a coleta da força muscular isocinética dos grupos extensor e flexor do joelho. Realizaram 2 minutos de alongamento ativo dos músculos a serem testados e 3 minutos de aquecimento na esteira ergométrica em velocidade não superior a 4 $\mathrm{km} / \mathrm{h}$. O protocolo de avaliação isocinética para o joelho foi realizado conforme recomendações propostas na literatura (DANNESKIOLD-SAMS $\varnothing \mathrm{E}$ et al., 2009; DVIR, 2002; MAUPAS et al., 2002; TERRERI et al., 2001): 5 repetições à velocidade angular de $60 \% \mathrm{~s}, 5$ repetições à velocidade angular de $180 \%$ s e 10 repetições à velocidade angular de $300 \%$ s, e repouso de 15 s entre as séries.

Para tanto, os indivíduos foram acomodados na cadeira do dinamômetro com inclinação do tronco de aproximadamente 80 e e coxas apoiadas no assento. 0 braço de alavanca do aparelho foi colocado paralelamente à perna com uma almofada fixada distalmente, alinhando o eixo do dinamômetro ao eixo do joelho. A estabilização na cadeira foi realizada por meio de cintos fixados no tórax, quadril e coxa do membro a ser testado. Em seguida, foi realizada correção da força da gravidade e peso do membro avaliado (PERRIN, 1993).

Os sujeitos foram incentivados verbalmente, antes e durante o teste no dinamômetro isocinético Biodex System 4 PRO, visando o encorajamento para realizar o teste com o maior empenho possível, extraindo a melhor performance muscular a cada repetição de cada série. Além disso, recebiam retorno visual da atividade muscular testada por meio do monitor acoplado ao dinamômetro. 
As variáveis de interesse estudadas foram: membro dominante, tempo de prática de corrida, torque máximo (PkT), trabalho total (Trab) e potência (Pot). Através do teste isocinético bilateral de joelho, foram coletadas as variáveis de deficiências de torque, trabalho e potência relacionadas aos músculos quadríceps e isquiotibiais entre os membros. De posse desses dados, foram calculados os Índices de Deficiência Muscular - IDMs. Para tanto, foi admitido como referencial de equilíbrio até $10 \%$ na diferença do cálculo entre os membros, sob a fórmula IDM=(PkT+Trab+Pot)/3. Os IDMs foram utilizados como referência para elaborar o desenho de equilíbrio muscular do joelho dos indivíduos envolvidos (SHINZATO; BATISTELLA, 1996; SHINZATO et al., 1996).

Os resultados das variáveis contínuas foram apresentados sob a forma de média, desvio padrão (DP) e mediana. As variáveis categóricas foram apresentadas sob a forma de percentual. Foi utilizado o software SPSS for Windows (versão 17.0) para tabulação e análise dos dados.

\section{RESULTADOS}

A amostra foi composta por uma maioria de adultos jovens do sexo masculino tendo o membro inferior direito como dominante. Com relação aos indicadores antropométricos e levando em consideração o índice de massa corporal (IMC), o cálculo amostral foi classificado em sua maioria como eutrófico (peso adequado conforme altura). O tempo de prática de corrida apresentou-se variável e pouco homogêneo entre os indivíduos estudados (Tabela 1).

Tabela 1 - Dados sociodemográficos e antropométricos dos corredores recreativos

\begin{tabular}{lrrrrr}
$\quad$ Característica & N & \% & Média & DP & Mediana \\
\hline Sexo & & & & & \\
$\quad$ Masculino & 68 & 61,3 & - & - & - \\
$\quad$ Feminino & 43 & 38,7 & - & - & - \\
Membro Dominante & & & & & \\
$\quad$ Direito & 100 & 90,1 & - & - & - \\
$\quad$ Esquerdo & 11 & 9,9 & - & - & - \\
Tempo de Prática de & - & - & 51,22 & 63,45 & 30,00 \\
Corrida (meses) & & & & & \\
Idade (anos) & - & - & 40,68 & 9,08 & 40,00 \\
Peso (kg) & - & - & 73,12 & 12,08 & 73,00 \\
\hline Altura (m) & - & - & 1,70 & 0,08 & 1,70 \\
IMC & - & - & 25,11 & 3,04 & 24,65 \\
\hline
\end{tabular}

Fonte: Autoria própria

Na comparação de força muscular dos joelhos entre o membro dominante e o não dominante, observou-se maiores forças, ainda que discretamente, em extensores no membro dominante e flexores no membro não dominante (Tabela 2).
Tabela 2 - Força muscular extensora e flexora de joelhos e dominância de membro

\begin{tabular}{lcc}
\hline \multicolumn{1}{c}{ Característica } & Frequência & $\%$ \\
\hline Maior Força Muscular Extensora de Joelho & & \\
Membro Dominante & 56 & 50,5 \\
Membro Não Dominante & 55 & 49,5 \\
Maior Força Muscular Flexora de Joelho & & \\
Membro Dominante & 52 & 46,8 \\
Membro Não Dominante & 59 & 53,2 \\
\hline
\end{tabular}

Fonte: Autoria própria

A relação de equilíbrio muscular entre os membros foi calculada levando-se em consideração as variáveis que compõem a fórmula do Índice de Deficiência Muscular (IDM). Após a avaliação isocinética e o cálculo do IDM, observou-se, também de forma discreta, um maior equilíbrio muscular entre extensores e uma relação de maior desequilíbrio muscular entre flexores de joelho (Tabela 3).

Tabela 3 - Relação de equilíbrio de joelho através do Índice de Deficiência Muscular

\begin{tabular}{llc}
\hline \multicolumn{1}{c}{ Característica } & Frequência & $\%$ \\
\hline Equilíbrio dos Extensores de Joelho através do IDM & & \\
$\quad$ Equilibrado (IDM $<10 \%)$ & 60 & 54,1 \\
Desequilibrado (IDM > 10\%) & 51 & 45,9 \\
Equilíbrio dos Flexores de Joelho através do IDM & & \\
Equilibrado (IDM $<10 \%)$ & 49 & 44,1 \\
Desequilibrado (IDM $>10 \%)$ & 62 & 55,9 \\
\hline
\end{tabular}

Fonte: Autoria própria

Não foi observada diferença relevante entre a força muscular de joelho apresentada pelos membros dominante e não dominante, nem tampouco na relação de equilíbrio/desequilíbrio através do IDM, possivelmente por se tratar de uma amostra pouco homogênea conforme tempo de prática esportiva dos corredores recreacionais.

\section{DISCUSSÃO}

O desequilíbrio muscular vem sendo investigado como um dos parâmetros desencadeadores de baixa performance esportiva de corredores. A investigação dessa relação de equilíbrio muscular sobre a articulação joelho é comum a despeito da sua ampla atuação na biomecânica de corrida. Para Bittencourt et al. (2005), devido às demandas esportivas, desequilíbrios entre as musculaturas extensora e flexora podem surgir, provocando sobrecarga estática ou dinâmica das estruturas musculotendíneas da articulação do joelho. Portanto, Ferreira et al. (2010) classificam a avaliação de déficits e desequilíbrios de força muscular como incontestável, principalmente pelo alto percentual de atletas jovens que já sofreram lesão.

No estudo de Ferreira et al. (2010), o quadríceps e os isquiotibiais, por serem músculos muito exigidos no esporte, necessitam de investigação tanto no membro dominante como no contralateral, a fim melhorar a 
programação dos treinamentos. Bittencourt et al. (2005) complementam que o desequilíbrio muscular da articulação do joelho não depende da dominância do membro, já que foi encontrada uma frequência de $53,7 \%$ de desequilíbrio no membro dominante e $49,3 \%$ no membro não dominante. Em recente estudo com vinte corredores, Saragiotto et al. (2016) apontaram uma associação de $21 \%$ envolvendo o desequilíbrio de força existente entre os músculos flexores e extensores de joelho como evento predisponente para o surgimento de lesões. Vale ressaltar que existe uma tendência de permanência de desequilíbrio muscular nos atletas lesionados, fato que contribui para o surgimento de uma nova lesão.

Em nossa casuística, valores próximos foram encontrados com extensores mais fortes no membro dominante em $50,5 \%$ e flexores mais fortes no membro não dominante em $53,2 \%$ da amostra. Isto reforça a ideia de que o fator dominância de membro não exerce uma influência significativa para o padrão de equilíbrio/desequilíbrio muscular sobre a articulação do joelho.

Outro estudo sobre os efeitos da dominância e fadiga do membro na biomecânica da corrida também revelou que a dominância de membros não afetou as diferenças cinemáticas ou cinéticas, indicando que a reabilitação pode ser exercida sem levar em consideração o domínio dos membros. Os autores desse estudo também apontam que os membros fadigam em taxa semelhante, independente da manifestação de dominância corporal (BROWN; ZIFCHOCK; HILLSTROM, 2014).

Quando analisamos as relações de equilíbrio ou desequilíbrio através do IDM no nosso estudo, encontramos $54,1 \%$ de equilíbrio extensor e $55,9 \%$ de desequilíbrio flexor. Tal achado sugere que praticamente metade da amostra se encontra numa situação de desequilíbrio com um maior predomínio na musculatura flexora. Tendo em vista que a utilização do IDM é um parâmetro muito confiável, conforme indicado no estudo de Shinzato et al. (1996), mensurar esse desequilíbrio muscular merece especial atenção devido à possibilidade de estar associado a uma lesão.

Uma possível hipótese para explicar a tendência de desequilíbrio flexor de joelho em corredores reside no fato de que essa modalidade esportiva induza uma menor participação da musculatura flexora à medida que sua velocidade de execução aumenta. Gazendam e Hof (2007) verificaram que a ativação da musculatura flexora de joeIho aumentou com o incremento de velocidade de corrida até alcançar $3,0 \mathrm{~m} . \mathrm{s}^{-1}$, reduzindo após esse referencial.

Portanto o grande valor da avaliação isocinética consiste na possibilidade de se quantificar os parâmetros musculares ao longo de processos preventivos, competitivos ou de reabilitação de forma extremamente objetiva. Pode-se, deste modo, direcionar o treinamento para superar deficiências específicas, buscando o equilíbrio entre os grupos musculares e estabelecendo metas para retorno e/ou melhora de performance no esporte (AQUINO et al., 2007; FREITAS et al., 1999; GOULART; DIAS; ALTIMARI,
2008; LUNA et al., 2015; PERRIN; ROBERTSON; RAY, 1987).

Este estudo nos permitiu ter uma noção preliminar do perfil de equilíbrio muscular do joelho dos corredores recreacionais estudados. Todavia não é possível fazer inferências para a população geral, uma vez que o desenho do estudo não atende a este propósito. Nosso estudo, entretanto, representa uma amostra significativa de praticantes de corrida no âmbito local, além do fato de estes corredores terem buscado esse método de avaliação visando melhorar as características individualizadas de treinamento. Desta forma, os resultados encontrados são válidos e podem ser utilizados para ganho na performance esportiva, apesar da evidente necessidade de estudos mais abrangentes com o objetivo de verificar o real impacto das relações de equilíbrio muscular para a ocorrência de lesões.

\section{CONCLUSÃO}

Esta pesquisa apresentou um perfil de equilíbrio muscular dos extensores e flexores de joelho dominante e não dominante em corredores recreacionais. Acredita-se que a dominância de membros não é um fator ligado ao desequilíbrio de forças no membro inferior. Este achado de desequilíbrio de força entre joelhos pode estar ligado à predisposição de lesão e deve orientar as equipes multiprofissionais de saúde a definir um trabalho preventivo de treinamento muscular e esportivo.

Sugerimos a realização de estudos mais abrangentes e com acompanhamento prospectivo, visando esclarecer se há relação entre os desequilíbrios encontrados através do IDM e a ocorrência de lesões nos praticantes de corrida.

\section{REFERÊNCIAS}

ALVES, V.L.S.; DUARTE JUNIOR, A. Fisioterapia nas lesões do esporte. São Paulo: Atheneu, 2014.

AQUINO, C.F. et al. A utilização da dinamometria isocinética nas ciências do esporte e reabilitação. Rev. bras. ciên. mov., São Paulo, v. 15, n. 1, p. 93-100, 2007.

BITTENCOURT, N. F. N. et al. Avaliação muscular isocinética da articulação do joelho em atletas das seleções brasileiras infanto e juvenil de voleibol masculino. Rev. bras. med. esporte, São Paulo, v.11, n. 6, nov./dez. 2005.

BROWN, A.M.; ZIFCHOCK, R.A.; HILLSTROM, H.J. The effects of limb dominance and fatigue on running biomechanics. Gait posture, Oxford, v. 39, p.915-919, 2014.

DANNESKIOLD-SAMS $\varnothing \mathrm{E}, \mathrm{B}$. et al. Isokinetic and isometric muscle strength in a healthy population with special reference to age and gender. Acta physiol. (Oxf), Oxford, v. 197, supl. 673, p.1-68, 2009.

DVIR, Z. Isocinética - avaliações musculares, interpretações e aplicações clínicas. São Paulo: Editora Manole, 2002.

FERREIRA, A.P. et al. Avaliação do desempenho isocinético da musculatura extensora e flexora do joelho de atletas de futsal em membro dominante e não dominante. Rev. bras. ciênc. esporte, Campinas, v.32, n.1, p.229-243, set. 2010 .

FREDERICSON, M.; MISRA, A. K. Epidemiology and aetiology of marathon running injuries. Sports med., Aukland, v.37, n.4-5, p. 437-439, 2007. 
FREITAS, C.R. et al. Estudo comparativo de torques isocinéticos em atletas dos XI jogos da juventude. In: CONGRESSO BRASILEIRO DE BIOMECÂNICA, 8., 1999, Florianopólis.

GAZENDAM, M. G. J.; HOF, A. L. Averaged EMG profiles in jogging and running at different speeds. Gait posture, Oxford, v. 25, p. 604-614, 2007.

GOULART, L.F.; DIAS, R.M.R.; ALTIMARI, L.R. Variação do equilíbrio muscular durante uma temporada em jogadores de futebol categoria sub-20. Rev. bras. med. esporte, São Paulo, v. 14, n.1, p. 17-21, 2008.

HINO, A. A .F. et al. Prevalência de lesões em corredores de rua e fatores associados. Rev. bras. med. esporte, São Paulo, v.15, n.1, jan./fev. 2009.

HRELJAC, A. Etiology, prevention, and early intervention of overuse injuries in runners: a biomechanical perspective. Phys. Med. Rehabil. Clin. N. Am., Estados Unidos, v.16, n. 3, p. 651-657, 2005.

LEPPÄNEN, M. et al. Interventions to prevent sports related injuries: a systematic review and meta-analysis of randomised controlled trials. Sports med., Auckland, v. 44, n.4, p.473-486, 2014.

LOPES, A.D. et al. What are the main running-related musculoskeletal injuries? A systematic review. Sports med., Auckland, v.42, n.10, p. 891-905, 2012.

LUNA, N.M.S. et al. Análise isocinética e cinética de corredores e triatletas com e sem histórico de fratura por estresse. Rev. bras. med. esporte, São Paulo, v. 21, n. 4, p. 252-256, 2015.

MAUPAS, E. et al. Functional asymmetries of the lower limbs. A comparison between clinical assessment of laterality, isokinetic evaluation and electrogoniometric monitoring of knees during walking. Gait posture, Oxford, v.16, n.3, p. 304-312, 2002.

PERRIN, D.H. Isokinetic exercise and assessment. Illinois: Champaign Human Kinetics Publishers, 1993.

PERRIN, D.H.; ROBERTSON, R.J.; RAY, R.L. Bilateral isokinetic peak torque, torque acceleration energy, power, and work relationships in athletes and nonathletes. J. orthop. sports phys. ther., Washington, v. 9, n. 5, p. 184-189, 1987.

PONCE, D. A. et al. Conceitualização e análise crítica dos dinamômetros isocinéticos. Braz. J. Biomec., São Paulo, v. 12, n. 23, p. 65-74, 2013.

SARAGIOTTO, B.T. et al. Desequilíbrio muscular dos flexores e extensores do joelho associado ao surgimento de lesão musculoesquelética relacionada à corrida: um estudo de coorte prospectivo. Rev. bras. ciênc. esporte, Campinas, v.38, n.1, p.64-68, 2016.

SARAGIOTTO, B. T et al. What are the main risk factors for runningrelated injuries? Sports med., Auckland, v. 44, n. 8, p. 1153-1163, 2014.

SHINZATO, G.T.; BATISTELLA, L.R. Exercício isocinético: sua utilização para avaliação e reabilitação músculo-esquelética. Âmbito Med Desportiva, [s.I], v.1, p.11-18, 1996.

SHINZATO, G.T. et al. Protocolo de avaliação funcional de joelho em patologias ortopédicas. Acta fisiátrica, São Paulo, v.3, n.1, p. 30-36, 1996.

SIQUEIRA, C.M. et al. Isokinetic dynamometry of knee flexors and extensors: comparative study among non-athletes, jumper athletes and runner athletes. Rev. Hosp. Clin. Fac. Med. São Paulo, São Paulo, v. 57, n.1, p. 19-24, 2002.

STRAKOWSKI, J.A.; JAMIL, T. Management of common running injuries. Phys. Med. Rehabil. Clin. N. Am., Estados Unidos, v.17, n.3, p. 537552, 2006.

SOUZA, T.R. et al. Pronação excessiva e varismos de pé e perna: relação com o desenvolvimento de patologias músculo-esqueléticas - revisão de literatura. Fisioter. Pesqui., São Paulo, v.18, n.1, p. 92-98, jan./mar. 2011.

TERRERI, A.S. et al. Avaliação isocinética no joelho do atleta. Rev. bras. med. esporte, São Paulo, v.7, n.5, p.170-174, 2001.

XAVEROVA, Z. et al. Isokinetic strength profile of elite female handball players. J. Hum. Kinet., Polônia, v.49, p.257-266, 2015.

Submetido em: 04/11/2019

Aceito em: 30/11/2019 